\title{
Bibliometric Analysis of Patient Navigation Model Applied in Colorectal Cancer Care Based on Web of Science
}

\author{
Wenwen Cao' ${ }^{1}$, Xiaoli Chen ${ }^{*}$, Liyuan Guo ${ }^{2}$, Xianbo Pei ${ }^{1}$ \\ ${ }^{1}$ School of Health Sciences, Wuhan University, Wuhan, China \\ ${ }^{2}$ Malden Catholic High School, Malden, MA, USA \\ Email: *345725755@qq.com
}

How to cite this paper: Cao, W.W., Chen, X.L., Guo, L.Y. and Pei, X.B. (2019) Bibliometric Analysis of Patient Navigation Model Applied in Colorectal Cancer Care Based on Web of Science. Journal of Biosciences and Medicines, 7, 1-11. https://doi.org/10.4236/jbm.2019.73001

Received: January 7, 2019

Accepted: March 1, 2019

Published: March 4, 2019

Copyright () 2019 by author(s) and Scientific Research Publishing Inc. This work is licensed under the Creative Commons Attribution International License (CC BY 4.0).

http://creativecommons.org/licenses/by/4.0/

\begin{abstract}
Background: The mortality rate of colorectal cancer in China is on the rise, which has exceeded the global average rate, thus making the care of colorectal cancer widely concerned. As a comprehensive nursing model, the patient navigation model has shown its effectiveness in improving the quality of life among patients with chronic diseases and reducing the shortage of current healthcare resources in some area. This study aims to identify the current status and dilemma of the patient navigation model applied in colorectal cancer care in China. Methods: Bibliometric method was used to analyze published articles regarding the application of patient navigation model in colorectal cancer care retrieved from the Web of Science database. Statistics were presented using visualization method processed by the Bibliographic Items Co-occurrence Matrix Builder (BICOMB) software. Results: 124 articles, from 2006 to 2018, covering 14 countries, involving 931 researchers, 356 institutions and 60 publications were obtained; American research papers are the largest components of the aforementioned articles; only one of them is written by Chinese authors. The 3 leading institutions in this domain are University OF Rochester, Ohio State University, Icahn School of Medicine at Mount Sinai, yet the distribution of authors is relatively decentralized. "Cancer" is the main journal that publishes the researches in this domain. Research hotspots are on "cancer prevention and screening", "health disparities", "economic evaluation", "patient navigators", and "ethnicity". Conclusion: In China, the research on the patient navigation is still in its infancy. Therefore, the Chinese government should provide more endorsement to this particular area and encourage the cooperation with other countries. China, having a long way to go, should further explore patient navigation on colorectal cancer care and establish a model that matches its cultural backgrounds.
\end{abstract}




\section{Keywords}

Bibliometric Analysis, Patient Navigation, Colorectal, Cancer, Web of Science

\section{Introduction}

The morbidity of colorectal cancer ranks third in all cancers worldwide, third in men and second in women [1]. The incidence of colorectal cancer ranks fifth in all cancer incidence rates in China and is currently increasing at a rate of $3.9 \%$ per year, which exceeds the international level of $2 \%$ [2]. Therefore, the burden of colorectal cancer in China is growing, which has caused widespread concern in society. Since the corresponding treatment of colorectal cancer, such as surgery, may cause permanent changes in patients' defecating manners, in this way, the abdominal stoma will lead to transformations in the self-image; also there are series of side effects caused by chemotherapy. The absence of a proper cancer care system will adversely impact both the physical and mental conditions of the colorectal cancer survivors, which lowers their quality of life. Thus, finding the cancer care model that fits the Chinese cultural background for colorectal cancer survivors is a problem that needs to be explored.

The patient navigation is defined as a model to guide patients to use the resources and services to meet their health needs, help them solve the barriers to timely cancer care, ameliorate the disease outcomes, and improve patient satisfaction and overall medical quality by Oncology Nursing Society [3]. Therefore, patient navigation model emerged in this particular environment [4]. It has been widely used in cancer care researches in many countries and regions, such as the United States and has shown its effectiveness. However, due to the differences in medical systems and cultural background between China and other countries, China has not established a systematic patient navigation model. Therefore, further understanding the content and role of the patient navigation model is needed to tailor a special patient navigation model that possesses the adaptability for Chinese culture.

Since the documents included in the Web of Sciences (WoS) database have higher academic value and are more representative of the international trend of discipline development; this study, based on WoS's core database, retrieves relevant professional statistics. Moreover, Bibliographic Items Co-occurrence Matrix Builder (BICOMB) software is used as a tool to comprehensively display this research's historical trajectory, current condition, and frontier of research, and to objectively analyze important authors, journals and core literatures. This research aims to provide reference for the academic research of patient navigational models of colorectal cancer patients in China.

\section{Research Methods}

\subsection{Statistical Sources}

Literature search was performed in May $10^{\text {th }}, 2018$ in the WoS core database, 
using the keywords ("patient navigation") AND (cancer* care*) AND (colorec$\left.\operatorname{tal}^{*}\right)$ without the limits of time frame $\left({ }^{*}\right.$ means that the searching result including the synonym of the word). The language was confined to English only, paper type were confined to articles or reviews. After the selection of the literature, 124 documents were retrieved. All records of the documents, including references, were exported in text format. The update time of the database is May 9, 2018.

\subsection{Research Tools}

This paper uses bibliometric method and utilizes BICOMB to research the quantity trend, distribution of countries and regions, research institutions, funding condition, document categories, influential documents, and frequent keywords regarding the patient navigation model applied in colorectal cancer. The BICOMB Indexes in this study include total articles in publication list and global citation times, which indicates the number of citation of a certain article in the WoS database.

\section{Result}

\subsection{General Information}

The 124 articles retrieved were all published in English. The document types are either articles or reviews.

\subsection{Publication Years and the Numbers of Citation}

The earliest document concerning the patient navigation model in colorectal cancer in WoS was published in 2006 by Jandorfin Cancer and was titled "Creating alliances to improve cancer prevention and detection among urban medically underserved minority groups-The East Harlem Partnership for Cancer Awareness" [5]. This study uses BICOMB to analyze the number of publications of the documents regarding the patient navigation model in colorectal cancer and imports relevant data into Excel to generate a composite chart. The result shows that the number of publications and global citation times have an ascending trend; the former has a gradual increase between 2010 and 2013 and soars after 2014. The number of publications from 2014 to 2017 is 2.2 times of that from 2006 to 2013. Furthermore, global citation times began to increase between 2008 and 2010 and showed a significant growth after 2010 . The global citation times of 2017 are nearly twice that of 2013, which is shown by Figure 1 .

\subsection{The Distribution of Countries/Regions}

The result displays that a total of 14 countries and regions have published articles regarding the application of patient navigation model in colorectal cancer in international journals. Moreover, Table 1 shows ten countries and regions with the most publications. According to the result, Western countries such as the United States and Canada have been exceedingly active in this domain and 
have published much more articles than the rest of countries/regions. The total publications of US and Canada account for $95 \%$ of all the articles. In addition, countries including France, Australia, and Iceland are the next in the list. Mainland China, along with other 9 countries, has only published 1 article, which accounts for $0.8 \%$ of the total publications.

\subsection{The Distribution of Institutions}

The distribution of the institutions that have published relevant articles was analyzed by statistical description, which reflects their scientific research ability and characteristics. The result of Table 2 indicates that the ten institutions with the most publications, which are all located in the United States; 9 of them are universities and their affiliates. The United States' Centers for Disease Control Prevention and the University of Colorado System both rank fifth in the number of publications.

\subsection{Funding Projects and the Distribution of Their Sources}

The statistical analysis of all the published documents reveals that 56 of them are funding projects. 47 of them were funded by National Cancer Institute. National Institute for Health Research and Centers for Disease Control and Prevention rank the second and third, respectively.

\subsection{The Distribution of Journals}

The statistical sorting of the journals indicates that a total of 60 journals have published articles that present findings about the patient navigation model in colorectal cancer care ten journals with the most publications are shown in $\mathrm{Ta}$ ble 3. Cancer, Cancer Epidemiology Biomarkers \& Prevention, and Journal of Cancer Education are the three major journals. Their numbers of publications are 19,9 , and 8 , which respectively accounts for $15 \%, 7 \%$, and $6.5 \%$ of the total value. Cancer has the highest number of publications and has an impact factor of 6.072 .

\subsection{Keywords and Hot Spot Analyze}

After combining and counting the synonyms in keywords, this paper utilizes the high and low word frequency boundary formula of the Second Law of Zipf [6] [7] and finds that the demarcation between high-frequency and low-frequency keywords is 13. With the removal of two keywords "patient navigation" and "colorectal cancer", the high-frequency words include cancer screening, health disparities, colonoscopy, and cancer prevention and control. The cancer screening, prevention and control are part of the whole cancer curriculum, but relatively early stages, followed by the diagnosis, treatment and survival period. The colonoscopy is the main method that people undergo the screening process. Health disparity, means the inequality access to medical resources through different economic, ethnic, and area groups (Table 4). 
Table 1. The distribution of countries/regions that published documents regarding the application of patient navigation model in colorectal cancer in WoS.

\begin{tabular}{cccc}
\hline Ranking & Countries/Regions & Publications & $\begin{array}{c}\text { Percentage of the } \\
\text { Total Publications (\%) }\end{array}$ \\
\hline 1 & The United States & 113 & 91.13 \\
2 & Canada & 5 & 4.03 \\
3 & France & 4 & 3.22 \\
4 & Australia & 2 & 1.61 \\
4 & Iceland & 2 & 1.61 \\
5 & The United Kingdom & 1 & 0.80 \\
5 & New Zealand & 1 & 0.80 \\
5 & Mainland China & 1 & 0.80 \\
5 & Spain & 1 & 0.80 \\
5 & Switzerland & 1 & 0.80 \\
5 & Oman & 1 & 0.80 \\
5 & Thailand & 1 & 0.80 \\
\hline
\end{tabular}

Note: Some articles were written by multiple countries, which causes overlaps in the numbers of publications in certain countries.

Table 2. The ten institutions with the most Publications about the Patient Navigation Model of Colorectal Cancer Care in WoS.

\begin{tabular}{|c|c|c|c|c|}
\hline Ranking & Institutions & Country & Publications & $\begin{array}{l}\text { Percentage of the } \\
\text { Total (\%) }\end{array}$ \\
\hline 1 & $\begin{array}{l}\text { University } \\
\text { of Rochester }\end{array}$ & US & 32 & $24.62 \%$ \\
\hline 2 & Ohio State University & US & 24 & $18.46 \%$ \\
\hline 3 & Icahn School of Medicine at Mount Sinai & US & 22 & $16.92 \%$ \\
\hline 4 & $\begin{array}{l}\text { University } \\
\text { of Texas System }\end{array}$ & US & 21 & $16.15 \%$ \\
\hline 5 & $\begin{array}{l}\text { University of } \\
\text { Colorado System }\end{array}$ & US & 19 & $14.62 \%$ \\
\hline 5 & Centers for Disease Control Prevention USA & US & 19 & $14.62 \%$ \\
\hline 7 & $\begin{array}{c}\text { University of } \\
\text { California System }\end{array}$ & US & 16 & $12.31 \%$ \\
\hline 7 & $\begin{array}{l}\text { University of } \\
\text { Washington }\end{array}$ & US & 16 & $12.31 \%$ \\
\hline 9 & Harvard University & US & 10 & $7.69 \%$ \\
\hline 9 & $\begin{array}{c}\text { University of Texas Health Science Center } \\
\text { Houston }\end{array}$ & US & 10 & $7.69 \%$ \\
\hline
\end{tabular}

Note: Some articles were written by multiple countries, which causes overlaps in the numbers of publications in certain countries. 
Table 3. The distribution of Journals.

\begin{tabular}{|c|c|c|c|c|c|}
\hline Ranking & Journal & $\begin{array}{l}\text { Frequency of } \\
\text { Occurrence }\end{array}$ & $\begin{array}{l}\text { Percentage } \\
(\%)\end{array}$ & $\begin{array}{c}\text { Cumulative } \\
\text { Percentage (\%) }\end{array}$ & $\begin{array}{l}\text { Impact } \\
\text { Factor IF }\end{array}$ \\
\hline 1 & Cancer & 19 & 15.32 & 15.32 & 6.072 \\
\hline 2 & $\begin{array}{l}\text { Cancer Epidemiology } \\
\text { Biomarkers \& Prevention }\end{array}$ & 9 & 7.26 & 22.58 & 4.142 \\
\hline 3 & Journal of Cancer Education & 8 & 6.45 & 29.03 & 1.329 \\
\hline 4 & Cancer Causes \& Control & 5 & 4.03 & 33.06 & 2.51 \\
\hline 5 & $\begin{array}{c}\text { Journal of General Internal } \\
\text { Medicine }\end{array}$ & 5 & 4.03 & 37.10 & 3.701 \\
\hline 6 & Supportive Care in Cancer & 4 & 3.23 & 40.32 & 2.698 \\
\hline 7 & $\begin{array}{l}\text { American Journal of } \\
\text { Gastroenterology }\end{array}$ & 4 & 3.23 & 43.55 & 9.566 \\
\hline 8 & $\begin{array}{l}\text { Journal of Health Care for } \\
\text { the Poor and Underserved }\end{array}$ & 3 & 2.4194 & 45.9677 & 0.916 \\
\hline 9 & Health Education \& Behavior & 3 & 2.4194 & 48.3871 & 1.808 \\
\hline 10 & BMC Cancer & 3 & 2.4194 & 53.2258 & 3.288 \\
\hline
\end{tabular}

Table 4. Analysis of Keywords' frequency.

\begin{tabular}{|c|c|c|c|c|}
\hline Ranking & Keywords & $\begin{array}{l}\text { Frequency of } \\
\text { Occurrence }\end{array}$ & $\begin{array}{c}\text { Percentage } \\
(\%)\end{array}$ & $\begin{array}{c}\text { Cumulative } \\
\text { Percentage (\%) }\end{array}$ \\
\hline 1 & $\begin{array}{c}\text { Cancer } \\
\text { Screening }\end{array}$ & 52 & 11.42 & 11.42 \\
\hline 2 & $\begin{array}{c}\text { Patient } \\
\text { Navigation }\end{array}$ & 41 & 9.01 & 20.43 \\
\hline 3 & $\begin{array}{l}\text { Colorectal } \\
\text { Cancer }\end{array}$ & 36 & 7.91 & 28.35 \\
\hline 4 & $\begin{array}{c}\text { Health } \\
\text { Disparities }\end{array}$ & 23 & 5.05 & 33.40 \\
\hline 5 & Colonoscopy & 20 & 4.39 & 37.80 \\
\hline 6 & $\begin{array}{l}\text { Cancer Prevention } \\
\text { and Control }\end{array}$ & 15 & 3.29 & 41.09 \\
\hline 7 & $\begin{array}{l}\text { Economic } \\
\text { Evaluation }\end{array}$ & 13 & 2.63 & 43.73 \\
\hline 8 & $\begin{array}{c}\text { Patient } \\
\text { Navigator }\end{array}$ & 11 & 2.41 & 46.15 \\
\hline 9 & Ethnicity & 8 & 1.75 & 47.91 \\
\hline 10 & $\begin{array}{l}\text { Breast } \\
\text { Cancer }\end{array}$ & 7 & 1.75 & 49.67 \\
\hline
\end{tabular}




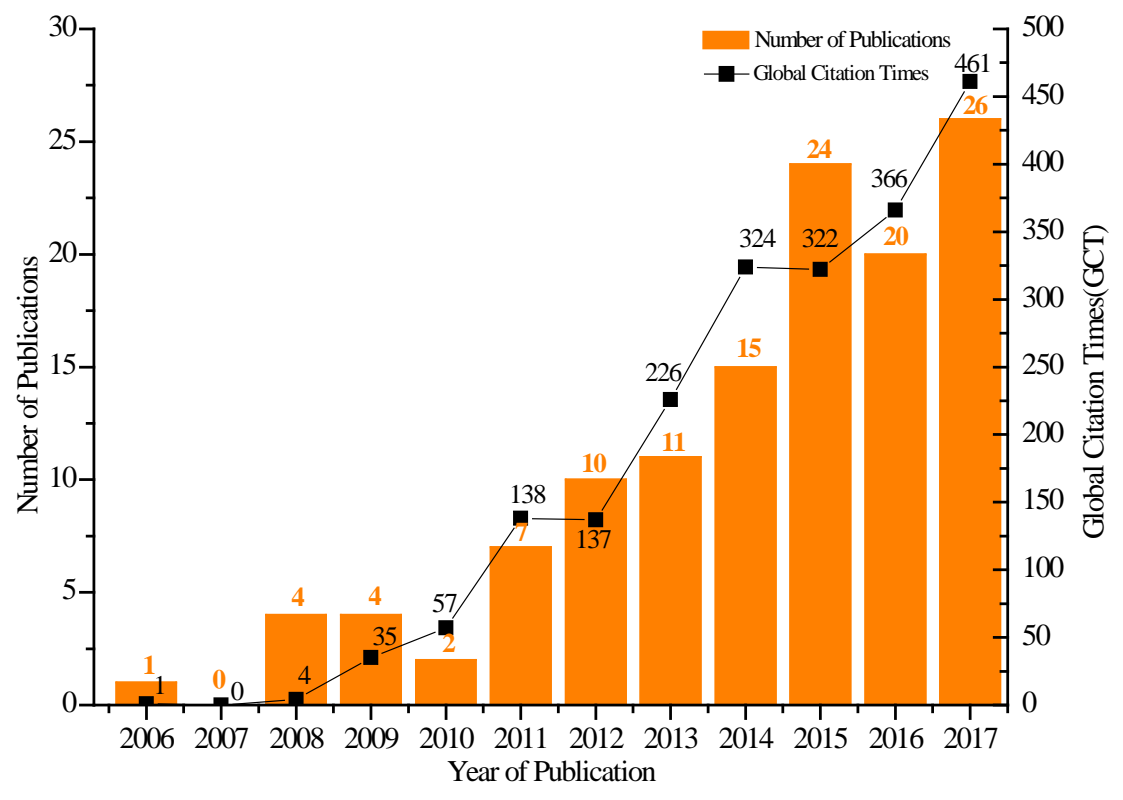

Figure 1. The time distribution of the documents regarding the application of patient navigation model in colorectal cancer in WoS.

\subsection{Articles with High Number of Citations}

"A Culturally Tailored Navigator Program for Colorectal Cancer Screening in a Community Health Center. A Randomized, Controlled Trial' Journal of General Internal Medicine, Percac-Lima, 2009 [8] has the highest number of citations: 44 times. The aforementioned paper exerts randomized controlled trials (RCT) to compare the adjusted patient navigation model with the regular care. After 9 months of carrying out controlled trials, the author finds the patient navigation model that is reasonably adjusted for the corresponding language and culture can significantly increase the screening rate of colorectal cancer for the low-income population and individuals who have distinct cultural and racial backgrounds.

\section{Discussion}

\subsection{The General Characteristics of the Included Articles}

Table 1 indicates that the earliest paper concerning the application of patient navigation model on colorectal cancer care was published in 2006 and was named Creating alliances to improve cancer prevention and detection among urban medically underserved minority groups-The East Harlem Partnership for Cancer Awareness [5], which is funded by the American Cancer Society. This paper is mainly about the successful implementation of the patient navigation model through the joint effort of individuals and organizations to alleviate inequalities in the screening of colorectal cancer. In addition, the total number of publications had a gradual increase trend between 2006 and 2018, especially after 2010, which is closely related to the overall development of the patient navigation model. The patient navigation model first developed in 1990, concentrated on female patients who were suffered from breast cancer and were at a 
disadvantage in medical care [9]. In 2005, the United States officially enacted the "Patient Navigation and Prevention of Chronic Infectious Diseases Act" to provide financial support for initiating patient navigation. The research on patient navigation was also supported by the Medicare subsidy service and the Centers for Disease Control [10]. Moreover, the Columbia Cancer Center of the United Kingdom first introduced a patient navigation model in 2005, and then the British researchers tested and improved the model from different perspectives [11].

\subsection{Enhancing Cooperation}

The distribution of the countries that have published relevant articles demonstrates that developed countries such as Europe and North America have far exceeded China in the patient navigation area. Among the 124 articles, one was written by multiple authors from China and the United States (Sun Yat-sen University cooperates with Johns Hopkins University). Comparing with the United States, China still needs development in terms of patient navigation model research. Therefore, China can conduct academic exchanges programs and cooperation with other countries such as the United States to promote the development of navigational care, also to import advanced scientific research methods and ideas, and thereby to find the patient navigation model suitable for various countries and regions that have different cultural backgrounds and developmental conditions.

\subsection{Encouraging Funding Endorsement}

The increasing trend of the number of publications and funding condition indicates that the development of research is closely related to financial support. The number of American publications and funded projects are both in the top position; in this domain, financial support can further accelerate developments. Therefore, financial support is indispensable for initiating a research in a certain area.

\section{Inspiration on China's Development of Patient Navigation Model}

\subsection{The Patient Navigation Model That Fit with China's Cultural Backgrounds Should Be Further Developed}

The popularity and quality of this research field are still in their infancy in China, which is notably different from that of the United States that can be seen from the country distribution part. This model has not been widely and effectively implemented in mainland China. However, the routine follow up care in some hospitals lays the foundation for conducting the patient navigation model, which proves the feasibility of the patient navigation model under China's environment. Inadequate primary medical resources, fear and distrust of existing medical condition, and complicated medical systems have significantly challenged the treatment and the rehabilitation of colorectal cancer, leading to the 
disparities in cancer outcomes [8], the disparity is also a research hot spot that was shown in the distribution of the word frequency. On this side, the program should be gradually extended to economically underdeveloped and rural areas where ethnic minorities live to provide culturally sensitive care for patients and improve their compliance for cancer treatment. In addition, China should initiate corresponding testing sites to study patients' existing barriers for medical care, further analyze the cost-effectiveness of the navigation model, and provide references for the national health policy makers, who will decide whether patient navigation should be included in medical insurance during the cancer treatment process.

\subsection{Role of Navigator Should Be Trained and Specified}

The role of navigator is also a research hot spot through the analysis. Not only who plays that role, but how could it be performed better matters. Navigators who match the patients' culture, abilities and language are one of the keys to ensure the viability and potential success of patient navigation. Various countries' studies indicate that not only nurses but also social workers, doctors, patients, and volunteers can work as navigators, but in mainland China, the follow up visits are all performed by nurses in the ward, thus making nurses have more chances to contact, accompany, and care for patients in the ward work, which renders the nurses an easier opportunity to find the obstacles faced by patients [12]. The training of navigators become necessary and should be thoroughly considered for its further implementation amid the China's setting.

\subsection{Patient Navigation Model Should Be Introduced into the China's Medical Industry, Especially the Nursing Field}

Few studies have introduced the concept of patient navigation into the nursing profession. Therefore, Chinese nursing researchers should contrive to learn from the relevant theories and applications of patient navigation and appropriately combine the characteristics of China's health policy and nursing profession in order to train hospitals and community nurses. In that way, patients will be provided with more timely and humanized care, the quality of care would also be improved, the disparities between cancer patients' outcomes will be reduced.

\section{Future Expectations}

Most of the current researches put their emphasis on the screening and prevention stage of cancer curriculum as it showed in the word frequency table. However, in a qualitative study, some patients expressed their will that the survivorship care can be taken into account [13]. Nonetheless, this article indicates that the patient navigation program lacks knowledge, funds and time to utilize in China [14]. Besides, developing patient navigation programs and improving the role of navigators must consider the main structure and environmental conditions of the organization. Thus, the roles and responsibilities of the navigator 
can reflect the needs of patients and organizations. Last but not least, the successful implementation of patient navigation model requires unanimous agreement, a certain clinical path, multidisciplinary training and collaboration. Relevant tutorials and resources for navigator training should be developed to facilitate the standardized training in patient navigation.

\section{Funding Project}

Ministry of Education of People's Republic of China (MOE), Humanities and Social Sciences Research Planning Fund Project (16YJAZH004).

\section{Conflicts of Interest}

The authors declare no conflicts of interest regarding the publication of this paper.

\section{References}

[1] WHO (2017) Fact Sheets. Cancer [EB/OL]. http://www.who.int/mediacentre/factsheets/fs297/en/

[2] Chen, W.Q., Zheng, R.S., Baade, P.D., et al. (2016) Cancer Statistics in China, 2015. CA: A Cancer Journal for Clinicians, 66, 115-132. https://doi.org/10.3322/caac.21338

[3] Smith, K., Efymow, B. and Gracia, C. (2012) Patient Navigation and Coordination of Care for the Oncofertility Patient: A Practical Guide. In: Gracia, C. and Woodruff, T.K., Eds., Oncofertility Medical Practice, Springer, New York, 175-185.

[4] Wells, K.J, Battaglia, T.A., Dudley, D.J., et al. (2008) Patient Navigation: State of the Art or Is It Science? Cancer, 113, 1999-2010. https://doi.org/10.1002/cncr.23815

[5] Jandorf, L., Fatone, A., Borker, P.V., et al. (2006) Creating Alliances to Improve Cancer Prevention and Detection among Urban Medically Underserved Minority Groups. The East Harlem Partnership for Cancer Awareness. Cancer, 107, 2043-2051. https://doi.org/10.1002/cncr.22153

[6] Sun, Q., Shaw, D. and Davis, C.H. (1999) A Model for Estimating the Occurrence of Same-Frequency Words and the Boundary between High- and Low-Frequency Words in Texts. Journal of the Association for Information Science \& Technology, 50, 280-286. https://doi.org/10.1002/(SICI)1097-4571(1999)50:3<280::AID-ASI11>3.0.CO;2-H

[7] Florence, P.S. and Zipf, G.K. (1950) Human Behaviour and the Principle of Least-Effort. Economic Journal, 60, 808-810. https://doi.org/10.2307/2226729

[8] Sanja, P.-L., et al. (2009) A Culturally Tailored Navigator Program for Colorectal Cancer Screening in a Community Health Center: A Randomized, Controlled Trial. Journal of General Internal Medicine, 24, 211-217. https://doi.org/10.1007/s11606-008-0864-x

[9] Freeman, H.P. and Rodriguez, R.L. (2011) History and Principles of Patient Navigation. Cancer, 117, 3537-3540. https://doi.org/10.1002/cncr.26262

[10] Freeman, H.P. (2013) The History, Principles, and Future of Patient Navigation: Commentary. Seminars in Oncology Nursing, 29, 72-75. https://doi.org/10.1016/j.soncn.2013.02.002

[11] Pedersen, A.E. and Hack, T.F. (2011) The British Columbia Patient Navigation 
Model: A Critical Analysis. Oncology Nursing Forum, 38, 200-206. https://doi.org/10.1188/11.ONF.200-206

[12] Ferrante, J.M., Cohen, D.J. and Crosson, J.C. (2010) Translating the Patient Navigator Approach to Meet the Needs of Primary Care. Journal of the American Board of Family Medicine, 23, 736-744. https://doi.org/10.3122/jabfm.2010.06.100085

[13] Davis, C., Darby, K., Likes, W., et al. (2009) Social Workers as Patient Navigators for Breast Cancer Survivors: What Do African-American Medically Underserved Women Think of This Idea? Social Work in Health Care, 48, 561-578. https://doi.org/10.1080/00981380902765212

[14] Malin, I. (2011) Oncology Nurses' Perspectives on the State of Cancer Survivorship Care: Current Practice and Barriers to Implementation. Oncology Nursing Forum, 38, 11-19. https://doi.org/10.1188/11.ONF.E11-E19 